\title{
Thermal stress state of rock massif with a spherical cavity taking into account inhomogeneity of the medium
}

\author{
Irina Frolova ${ }^{1}$, Vladimir Andreev ${ }^{1, *}$ \\ ${ }^{1}$ Moccow State University of Civil Engineering, Yaroslavskoye sh., Moscow 129110, Russia
}

\begin{abstract}
The case of continuous one-dimensional heterogeneity of deformation characteristics of a material of the pedigree array with the spherical cavity, received by means of explosion, are continuous functions of one of co-ordinates - radius is considered. Solution of an initial set of equations concerning displacements is sought in the form of decomposition in Fourier series in Legendre polynomials. Using the method of separation of variables, we can reduce the problem to a system of two ordinary differential equations with variable coefficients, which can be solved numerically. Stress analysis was performed in the homogeneous and inhomogeneous array surrounding the spherical cavity. A comparative analysis of the results is carried out.
\end{abstract}

\section{State of the problem}

The paper deals with the problem of elasticity theory in spherical coordinates with the combined effect on the inhomogeneous array with a cavity asymmetrical load (soil resistance) and centrally symmetric temperature field.

Calculation scheme of the problem is shown in Fig. 1.

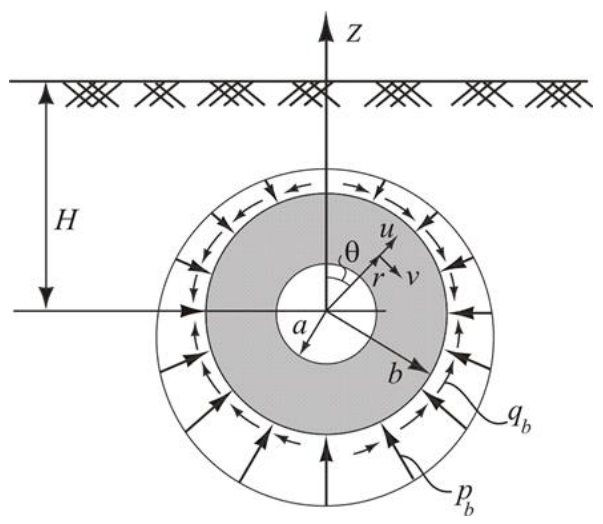

Fig. 1. Calculation scheme.

* Corresponding author: asv@,mgsu.ru 
Considered rock massif containing the spherical cavity obtained by explosion. When creating underground cavities by explosion releases large amount of heat in the cavity, thus there is a significant warming near a cavity array area with the subsequent distribution of heat in depths of the array and the cooling surface of the cavity [1]. This process is nonstationary, but excluding the initial period and given the relatively slow redistribution thermal field for a sufficiently long time later, the problem of determining the temperature stress can be regarded as quasistationary [2-5]. The calculation was made according the real working conditions of the array (the presence of ground resistance), and depending the mechanical characteristics of the soil on the temperature and on the fracturing of the array.

\section{Inhomogeneity of ground}

As is known $[1,6]$ using the method of forming explosive underground cavities, as well as drilling and blasting method for tunneling holes surrounding rock mass undergoing such changes (appearance of microcracks, compaction, sintering) that lead to mechanical inhomogeneity of material. At the same time there is local variation of the modulus of elasticity, which has a central symmetry. To describe the dependence E(r) can be used function obtained on the basis experimental results [7]:

$$
E(r)=E^{*}\left[1+\left(k_{1}-1\right)\left(\frac{a}{r}\right)^{m}\right],
$$

where $\mathrm{k} 1$ and $\mathrm{m}$ - empirical coefficients, $\mathrm{r}$ - the cavity radius, $\mathrm{E}^{*}$ - elasticity modulus of undisturbed array.

In solving the problem of thermoelasticity for large temperature gradients should take into account the dependence $\mathrm{E}^{*}(\mathrm{~T})$, which can be taken in the form:

$$
E^{*}=E_{0} e^{-\delta T}
$$

Here E0 - the value of the elastic modulus at $\mathrm{T}=0^{\circ} \mathrm{C}, \delta$ - empirical parameter. Such whole dependence $\mathrm{E}(\mathrm{r})$ has the form:

$$
E(r)=E_{0} e^{-\delta T(r)}\left[1+\left(k_{1}-1\right)\left(\frac{a}{r}\right)^{m}\right]
$$

\section{Solution of the problem}

To assess the stress state of the array under the influence of temperature field and external loads, you can use the equilibrium equations in displacements [8]:

$$
\begin{gathered}
\mu \nabla^{2} u+3(\lambda+\mu) \frac{\partial \varepsilon_{a v}}{\partial r}-\frac{2 \mu}{r^{2}}\left(u+\frac{\partial v}{\partial \theta}+v \operatorname{ctg} \theta\right)+3 \varepsilon_{a v} \frac{\partial \lambda}{\partial r}+2 \frac{\partial \mu}{\partial r} \frac{\partial u}{\partial r}+ \\
+\frac{1}{r} \frac{\partial \mu}{\partial \theta}\left(\frac{1}{r} \frac{\partial u}{\partial \theta}+\frac{\partial v}{\partial r}-\frac{v}{r}\right)-3 \frac{\partial}{\partial r}\left(K \varepsilon_{f}\right)=0 \\
\mu \nabla^{2} v+3 \frac{(\lambda+\mu)}{r} \frac{\partial \varepsilon_{\mathrm{av}}}{\partial \theta}+\frac{\mu}{r^{2}}\left(2 \frac{\partial u}{\partial \theta}-\frac{1}{\sin ^{2} \theta} v\right)+\frac{3}{r} \frac{\partial \lambda}{\partial \theta} \varepsilon_{a v}+\frac{\partial \mu}{\partial r}\left(\frac{1}{r} \frac{\partial u}{\partial \theta}+\frac{\partial v}{\partial r}-\frac{v}{r}\right)+ \\
+\frac{2}{r^{2}} \frac{\partial \mu}{\partial \theta}\left(u+\frac{\partial v}{\partial \theta}\right)-\frac{3}{r} \frac{\partial}{\partial \theta}\left(K \varepsilon_{f}\right)=0 .
\end{gathered}
$$


Here $\lambda(\mathrm{r}), \mu(\mathrm{r})$ - Lame constants, $K(r)$ - modulus of dilatation, $\varepsilon_{f}(r)=\alpha T(r)-$ forced temperature deformations; $\alpha=$ const - coefficient of linear thermal expansion; $v-$ Poisson's ratio; $\varepsilon_{a v}=\frac{1}{3}\left(\frac{\partial u}{\partial r}+\frac{1}{r} \frac{\partial v}{\partial \theta}+\frac{2 u}{r}+\frac{v}{r} \operatorname{ctg} \theta\right)-$ average strain.

The boundary conditions are:

$$
\begin{aligned}
& \text { on the inner surface }(\mathrm{r}=\mathrm{a}) \sigma_{r}=0, \tau_{r \theta}=0 ; \\
& \text { on the outer surface }(\mathrm{r}=\mathrm{b}) \quad \sigma_{r}=-p_{b}(\theta), \tau_{r \theta}=q_{b}(\theta),
\end{aligned}
$$

where $p_{b}$ and $q_{b}$ - surface loads (Fig. 1):

$$
p_{b}(\theta)=\gamma(H-b \cos \theta)\left(\frac{v}{1-v}+\frac{1-2 v}{1-v} \cos ^{2} \theta\right), \quad q_{b}(\theta)=\frac{1}{2} \gamma(H-b \cos \theta) \frac{1-2 v}{1-v} \sin 2 \theta
$$

The solution of system (3) with the boundary conditions (3) is invited to seek in the form displacement of expansions in Fourier series:

$$
u(r, \theta)=\sum_{n=0}^{\infty} u_{n}(r) P_{n}(\cos \theta), \quad v(r, \theta)=\sum_{n=0}^{\infty} v_{n}(r) \frac{d P_{n}(\cos \theta)}{d \theta} .
$$

where $P_{n}(\cos \theta)$ - Legendre polynomial of $n$-th degree.

Substituting the expressions (5) to the first equation (3) lead to the equation for unknown $u_{0}$ :

$$
u_{0}^{\prime \prime}(\lambda+2 \mu)+u_{0}^{\prime}\left[\frac{2(\lambda+2 \mu)}{r}+\lambda^{\prime}+2 \mu^{\prime}\right]-u_{0}\left[\frac{2(\lambda+2 \mu)}{r^{2}}-\frac{2}{r} \lambda^{\prime}\right]-3\left(K \varepsilon_{f}\right)^{\prime}=0
$$

and for $u_{n}$ and $v_{n}(n \geq 1)$ to a system of two ordinary differential equations of second order with variable coefficients:

$$
\begin{gathered}
u_{n}^{\prime \prime}(\lambda+2 \mu)+u_{n}^{\prime}\left[\frac{2(\lambda+2 \mu)}{r}+\lambda^{\prime}+2 \mu^{\prime}\right]-u_{n}\left[\frac{\mu}{r^{2}} n(n+1)+\frac{2(\lambda+2 \mu)}{r^{2}}-\frac{2}{r} \lambda^{\prime}\right]+ \\
+\left[-v_{n}^{\prime}\left(\frac{\lambda+\mu}{r}\right)+v_{n}\left(\frac{\lambda+3 \mu}{r^{2}}-\frac{1}{r} \lambda^{\prime}\right)\right] n(n+1)=0 ; \\
v_{n}^{\prime \prime} \mu+v_{n}^{\prime}\left(\frac{2 \mu}{r}+\mu^{\prime}\right)-v_{n}\left[n(n+1) \frac{\lambda+2 \mu}{r}+\frac{1}{r} \mu^{\prime}\right]+u_{n}^{\prime} \frac{\lambda+\mu}{r}+u_{n}\left[\frac{2(\lambda+2 \mu)}{r^{2}}+\frac{1}{r} \mu^{\prime}\right]=0 .
\end{gathered}
$$

Expanding the surface loads $p_{6}(\theta)$ and $q_{b}(\theta)$ (5) in series similar to (6), it is sufficient to restrict members to $n=3$ :

$$
p_{b}(\theta)=\sum_{n=0}^{3} c_{n} P_{n}(\cos \theta), \quad q_{b}(\theta)=\sum_{n=1}^{3} d_{n} \frac{d P_{n}(\cos \theta)}{d \theta} .
$$

The coefficients of these expansions $c_{n}$ and $d_{n}$ are determined by the known formulas [9]. Thus, systems (8) are solved for $n=0-3$.

The solution of system (8) for arbitrary dependencies $\lambda(r), \mu(r)$ and $K(r)$ should be carried out numerically. There are different ways of numerical realization of the considered equations. In this paper, method of the orthogonal matrix sweep is used [10]. 
After finding $u_{n}$ and $v_{n}$, we can calculate stresses $\sigma_{r}, \sigma_{\theta}, \sigma_{\varphi}$ and $\tau_{r \theta}$ according to the formulas:

$$
\begin{aligned}
\sigma_{r} & =\sum_{n=0}^{3}\left[\frac{2 \lambda}{r} u_{n}+(\lambda+2 \mu) u_{n}^{\prime}-\frac{\lambda n(n+1)}{r} v_{n}-3 K g_{n}\right] P_{n}(\cos \theta) \\
\sigma_{\theta} & =\sum_{n=0}^{3}\left\{\left[\frac{2(\lambda+2 \mu)}{r} u_{n}+\lambda u_{n}^{\prime}-\frac{\lambda n(n+1)}{r} v_{n}-3 K g_{n}\right] P_{n}(\cos \theta)+\frac{2 \mu}{r} v_{n} \frac{d^{2} P_{n}(\cos \theta)}{d \theta^{2}}\right\} \\
\sigma_{\varphi} & =\sum_{n=0}^{3}\left\{\left[\frac{2(\lambda+2 \mu)}{r} u_{n}+\lambda u_{n}^{\prime}-\frac{\lambda n(n+1)}{r} v_{n}-3 K g_{n}\right] P_{n}(\cos \theta)+\frac{2 \mu}{r} v_{n} \operatorname{ctg} \theta \frac{d P_{n}(\cos \theta)}{d \theta}\right\} ; \\
\tau_{r \theta} & =\sum_{n=1}^{3} \mu\left[\frac{1}{r} u_{n}-\frac{1}{r} v_{n}+v_{n}^{\prime}\right] \frac{d P_{n}(\cos \theta)}{d \theta}
\end{aligned}
$$

where $g_{0}=\alpha T(r)$ at $n=0, g_{n}=0$ at $n \neq 0$.

Calculations were carried out under the following initial data: $a=25 \mathrm{~m} ; b=250 \mathrm{~m}$; $H=1200 \mathrm{~m} ; \gamma=2.1510^{3} \mathrm{~kg} / \mathrm{m}^{3} ; E_{0}=210^{4} \mathrm{MPa} ; \mathrm{v}=0.23$.

Distribution of temperature in an isotropic medium under the influence of instantaneous heat sources distributed on the sphere of radius $a$ described by the formula [11]:

$$
T(r, t)=\frac{Q_{0}}{8 \pi \sqrt{\pi} c \gamma a r \sqrt{a_{1} t}} e^{-\frac{(r-a)^{2}}{4 a_{1} t}}+T_{1}
$$

Here $T_{1}$ - initial temperature of massif, $T$ - temperature at a point spaced from the center of the cavity by a distance $r \geq a$ at a time t; $Q_{0}$ amount of heat generated; c specific heat capacity of the environment; $\gamma_{-}$density; $a_{1}=\lambda / \mathrm{c} \gamma-$ temperature conductivity; $\lambda$ - thermal conductivity of the material; a - cavity radius.

\section{Results}

In the calculations used the averaged thermal characteristics of the soil: $c=0.96 \mathrm{~kJ} /(\mathrm{kg}$. deg.); $a_{1}=1.6710^{-6} \mathrm{~m}^{2} / \mathrm{s}$.

Fig. 2 shows the temperature curves for some moments in time $t$.

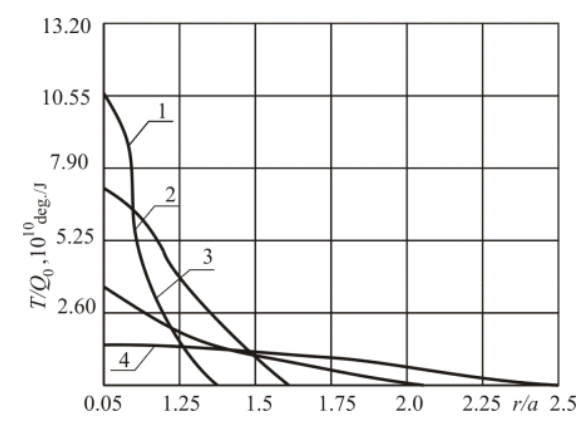

Fig.2. Temperature curves for different time.

$1-3.6 \cdot 10^{6} \mathrm{sec}$.; $2-7.2 \cdot 10^{6} \mathrm{sec}$.; $3-4.025 \cdot 10^{7} \mathrm{sec}$.; $4-1.61 \cdot 10^{8} \mathrm{sec}$.

Of considerable interest is the study of influence temperature inhomogeneity on the value of the components of the temperature stress state of the rock mass. Fig. 3 shows 
diagrams of thermal stresses $\sigma_{\theta}$ at time $t=1.61 \cdot 10^{8} \mathrm{sec}$. The parameter characterizing the fractured rock is equal $k_{1}=1$. The graph shows a significant change in the stresses near the contour in an inhomogeneous material as compared with the homogeneous that is associated with a significant drop in the modulus of elasticity due to temperature inhomogeneity.

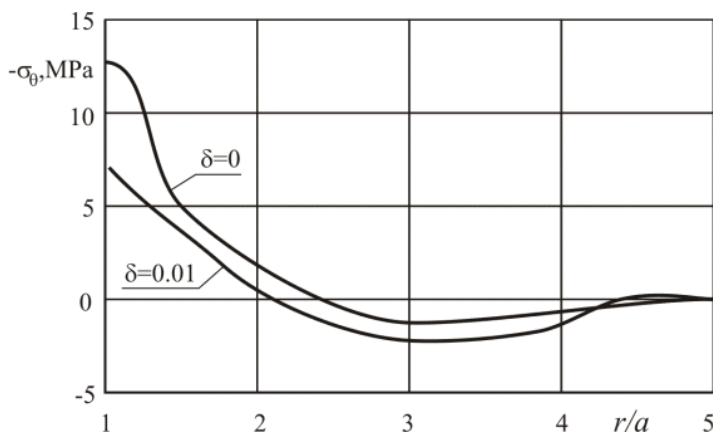

Fig. 3. Diagrams of thermal stresses. $\theta=90^{\circ} \mathrm{C} ; t=1.61 \cdot 10^{8} \mathrm{sec}$.; $k_{1}=1$.

Fig. 4 shows diagrams of the total stresses $\sigma_{\theta}\left(\right.$ at $\left.\theta=90^{\circ} C\right)$, caused by the simultaneous action of temperature and power load on the rock mass taking into account the inhomogeneity both types of material.

Changes stresses $\sigma_{\theta}$ along the contour of the cavity shown in Fig. 5

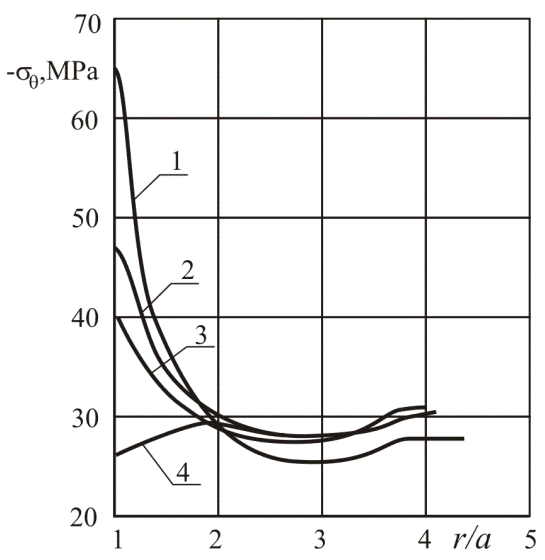

Fig. 4. Diagrams of the total stresses. $\sigma_{\theta}\left(\theta=90^{\circ} \mathrm{C} ; t=1.61 \cdot 10^{8} \mathrm{sec}.\right)$.

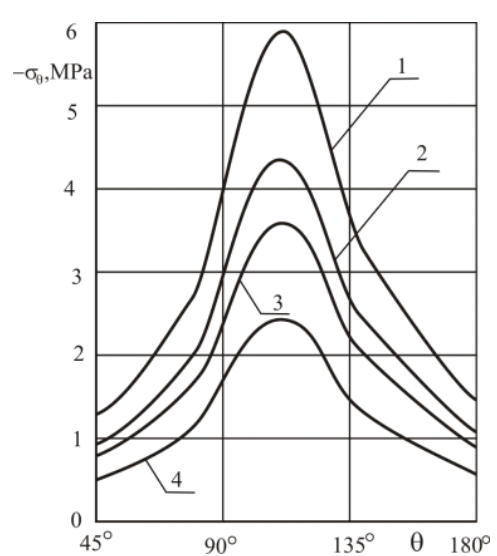

Fig. 5. Changes stresses $\sigma_{\theta}$ along the contour. $\left(t=1.61 \cdot 10^{8} \mathrm{sec}\right.$.).

$$
1-k_{1}=1, \delta=0 ; 2-k_{1}=1, \delta=0.01 ; 3-k_{1}=0.5, \delta=0 ; 4-k_{1}=0.5, \delta=0.01 \text {. }
$$

\section{Conclusions}

The Figures 3 and 4 show that allowance for two types of inhomogeneity leads to a more significant reduction the stresses, particularly in immediate vicinity of the cavity contour. Here, this stresses difference is about $50 \%$. Further away from the cavity contour at a distance equal to $2 a$ calculation results in an inhomogeneous and homogeneous cases virtually identical. 
Fig. 5 shows that the stresses differ greatly across the range of values for homogeneous and inhomogeneous arrays. Exceptions are zones that are close to the poles of the cavity ( and ). The biggest difference in the stresses values observed at . But when approaching the poles difference is becoming less marked.

Analyzing the results of the calculations, we can conclude that allowance for the inhomogeneity of the material is necessary in the calculation rock masses as at the power loads as well at temperature influences.

\section{Acknowledgment}

This work was financially supported by the Ministry of Russian Education (state task \#2014/14).

\section{References}

1. A. A. Bakirov, Application of underground nuclear explosions in the oil industry, (1981)

2. V. I. Andreev, A. S. Avershyev, Advanced Materials Research 838-841, 254-258 (2014)

3. V. I. Andreev, A. S. Avershyev, Procedia Engineering 111, 30-35 (2015)

4. V. I. Andreev, A. S. Avershyev, Structural mechanics of engineering structures and buildings 1, 30-37 (2014)

5. I. I. Frolova, Scientific Review 12, 94-99 (2015)

6. D. Rawson, P. Randolf, C. Boardman, V. Wheeler, J. of Geophysical Research 71 14, 3415-3426 (1966)

7. I. V. Baklashov, B. A. Kartozia, Rock mechanics, (1975)

8. A. I. Lurie, The theory of elasticity, (1970)

9. P. I. Romanovsky, Fourier series. Field theory, (1973)

10. A. A. Samarskiy, E. S. Nikolaev, Methods for solving grid equations, (1978)

11. A. V. Lykov, Heat conduction theory, (1967) 